\title{
MRI Signal Abnormalities of the Optic Tracts, a Marker of Meningoencephalitis Caused by Trypanosoma Gambiense (TG)-A Delayed Patho-Radlological Correlation
}

\author{
Cherif Mohamadou Aidara ${ }^{*}$, Caroline Magne ${ }^{2}$, Nfally Badji ${ }^{3}$, Lera Geraud Akpo ${ }^{3}$, \\ Gaelle Ebinda Mipinda ${ }^{4}$, Hamidou Deme ${ }^{3}$, Philomène Kouna², Abdoulaye Dione Diop5, \\ Abdoulaye Ndoye Diop5 ${ }^{5}$, Sokhna Ba ${ }^{5}$, Elhadj Niang3
}

${ }^{1}$ Radiology Office of "De la Paix University Hospital”, Ziguinchor, Senegal

${ }^{2}$ Internal Medicine Office of Polyclinique El Rapha, Libreville, Gabon

${ }^{3}$ Radiology Office of Aristide Le Dantec University Hospital, Dakar, Senegal

${ }^{4}$ Radiology Office of Polyclinique El Rapha, Libreville, Gabon

${ }^{5}$ Radiology Office of Fann University Hospital, Dakar, Senegal

Email: ^cfm.aidara@univ-zig.sn, ^matoouz@gmail.com

How to cite this paper: Aidara, C.M., Magne, C., Badji, N., Akpo, L.G., Mipinda, G.E., Deme, H., Kouna, P., Diop, A.D., Diop, A.N., Ba, S. and Niang, E. (2022) MRI Signal Abnormalities of the Optic Tracts, a Marker of Meningoencephalitis Caused by Trypanosoma Gambiense (TG) - A Delayed PathoRadlological Correlation. Open Journal of Radiology, 12, 1-7.

https://doi.org/10.4236/ojrad.2022.121001

Received: August 27, 2021

Accepted: January 3, 2022

Published: January 6, 2022

Copyright $\odot 2022$ by author(s) and Scientific Research Publishing Inc. This work is licensed under the Creative Commons Attribution International License (CC BY 4.0).

http://creativecommons.org/licenses/by/4.0/

\begin{abstract}
Parasitic meningoencephalitis presents several etiologies which sometimes depend on their geographical location. They require thorough blood and cerebrospinal fluid check-up for directing an efficient treatment. Clinicians and radiologists are constantly looking for specific signs that could point to a particular etiology. The meningoencephalitis caused by Human African Trypanosomiasis (HAT) due to Trypanosoma brucei gambiense (TG) is a rare disease characterized by a slow progression, over years sometimes. Its non-specific presentation either clinically or in imaging can lead to misdiagnosis and thus, delay the treatment. However, involvement of the optic tracts seems to be characteristic of this condition, on old data from animal experimentation and recent high-field MRI data. MRI is the best current technique to explore the brain, cranial nerves, and visual pathways. In this article, we are going to present two observations of meningoencephalitis caused by HAT and then discuss some specific aspects of this neglected and re-emerging disease.
\end{abstract}

\section{Keywords}

Human African Trypanosomiasis, Trypanosoma Brucei Gambiense, Diffuse Meningo-Encephalitis, Optic Tract Evolvement 


\section{Introduction}

HAT is a specific African parasitic disease caused by two flagellated protozoans. They are transmitted to human beings through the tsetse fly bites. The African continent over latitudes $15^{\circ}$ north and $20^{\circ}$ south offers ideal conditions for the development of this fly. Trypanosoma brucei gambiense (TG) is found in West and Central Africa while Trypanosoma brucei rhodesiense (TR) is found in East and South Africa (Figure 1). HAT due to TG progresses slowly, over years in some situations and represents almost $98 \%$ of all HAT cases. The disease follows two stages: the hemolymphatic stage and the late encephalic stage when the cerebrospinal fluid and the brain are involved. During this latest stage, sleep disturbances appear, hence the name "sleeping sickness".

The eradication program of the WHO is bearing fruit, as a result from 1998 to 2008 , the number of cases fell from more than 25,000 to more than 10,000 [1] [2]. The conclusive diagnosis is based on the detection of the parasite in the blood or the CSF and the presence of antiparasitic antibodies in the blood.

For this disease, medical imaging is non-specific and mainly shows signs of encephalitis in the meningoencephalitis stage [3]. The availability of neuroimaging tools leads to the exploration of patients suffering from meningoencephalitis. Its cause must be investigated for optimal treatment. Also, drug administration during the treatment such as melarsoprol in the meningoencephalitis stage is not without risk, linked to fatal arsenical encephalitis in 5\% [2].

Few experimental data are available on HAT due to TG. They were carried out by Bogaert in 1962 on cats in a period prior to the invention of the scanner and MRI tools [4] [5].

In this article, we are going to present two observations of HAT at the meningoencephalitis stage investigated on MRI and discuss some aspects of this disease and the correlation with the histopathological data.

\section{Cases Presentation}

\subsection{Observation 1}

Ms. O.H.C, 40 years old, presented the following symptoms: headache, slight fever with alertness disorder, nocturnal insomnia and diurnal somnolence. A left hemiparesis and an extrapyramidal syndrome were noted. MRI was performed on a high-field 1.5T siemens in the 3 planes with diffusion, T1 SE, T2 SE, T2 FLAIR, $\mathrm{T} 2{ }^{\star}$ and $\mathrm{T} 1$ gadolinium sequences. Blood tests lumbar puncture showed evidence of trypanosomiasis.

- MRI findings

MRI showed vasogenic cerebral edema predominantly in the gray nuclei and the right semioval center. We noted an involvement of the optic tract especially the right one that appeared thickened and with hypersignal. There was no significant enhancement despite the diffuse inflammation (Figure 2). 

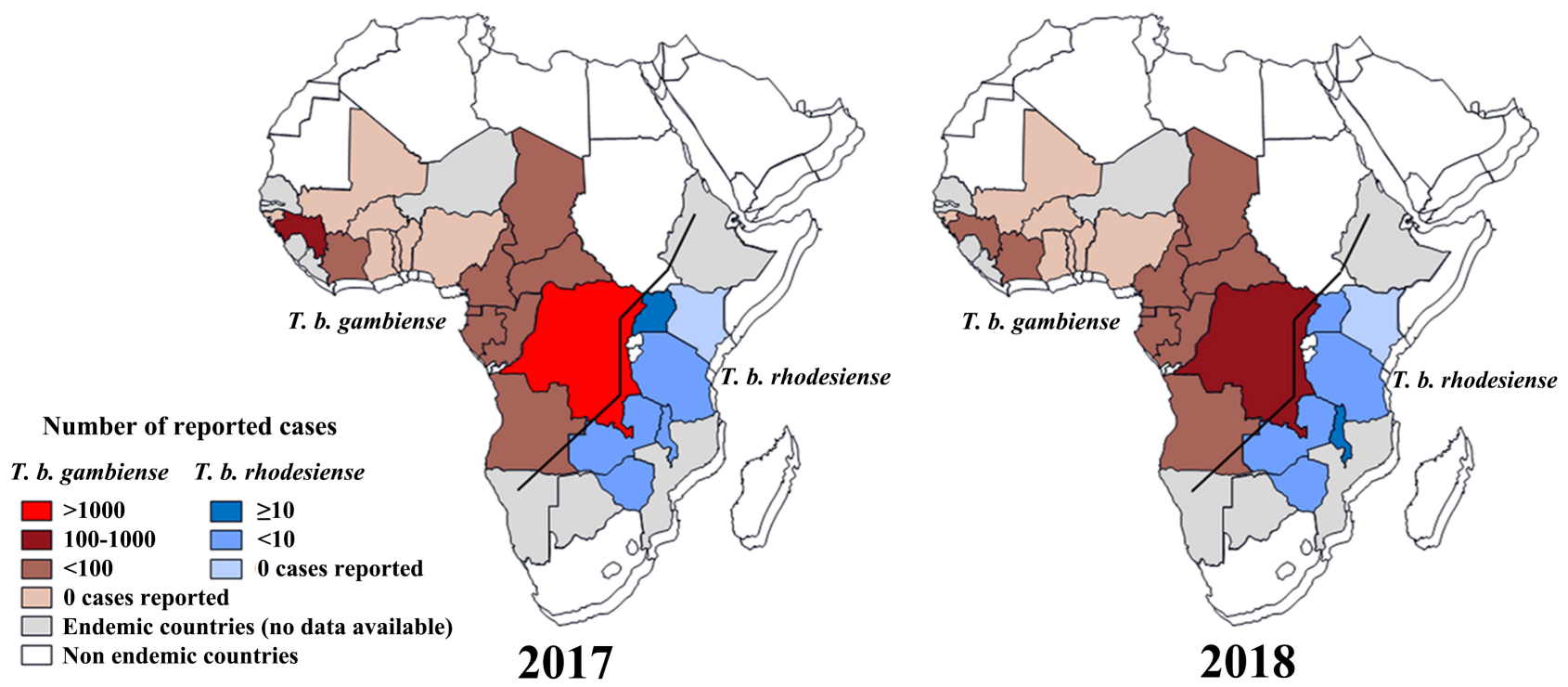

Figure 1. Showing the distribution of HAT [1].

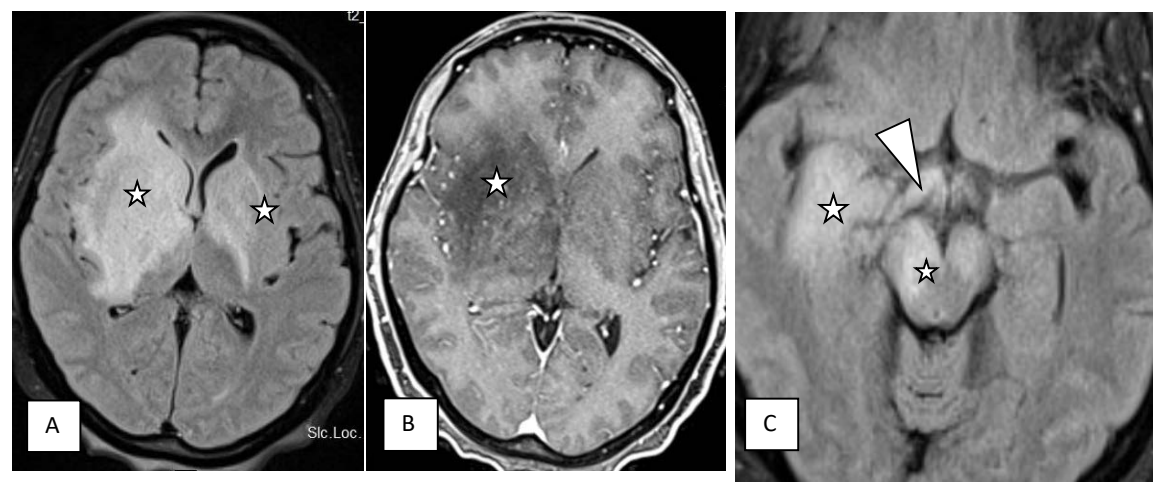

Figure 2. MRI axial section T2 FLAIR on A, and a T1SE after gadolinium on B. Extended edema on the basal ganglia and the white matter of the cerebrum, and the brainstem (stars in (A), (B) and (C)). Involvement of the optic tracts predominantly the right one that appear enlarged and hypersignal (arrowhead in $(C))$.

\section{- Diagnostic}

Encephalic form (stage 2) of human African trypanosomiasis with associated optic tracts involvement.

\subsection{Observation 2}

Mr. M.K, 45 years old, in the emergency room presented an alteration of consciousness associated with a tremor, abnormal somnolence without fever. A prior high field MRI check-up was done with the same protocol. Blood and cerebrospinal fluid identified evidence of trypanosomiasis.

\section{- MRI findings}

MRI showed a diffuse vasogenic edema in the grei nuclei and the white matter with a hypersignal in T2 sequences without gadolinium enhancement. Inflammatory signs with a hypersignal in T2 SE sequence and a slight enlargement of the optic tracts were noted (Figure 3). 


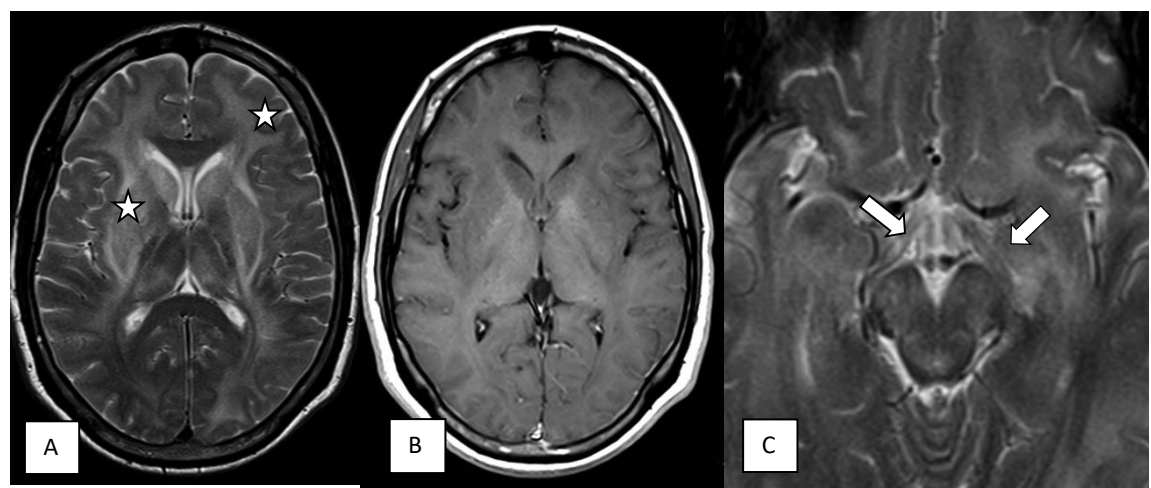

Figure 3. MRI axial sections T2 weighted (A) showing a diffuse edema of the white matter of the semioval centers and the basal ganglia (stars in (A)) without contrast gadolinium enhancement (B). Axial section T2SE (C) showing the involvement (hypersignal) of the optic tracts (arrow C).

\section{- Diagnostic}

Encephalic form (stage 2) of human African trypanosomiasis with associated optic tracts involvement.

\section{Comments}

\subsection{Focus Current Point of This Condition}

The sleeping sickness is caused by 2 parasites; Trypanosoma brucei gambiense (TG) found in West and Central Africa and Trypanosoma brucei rhodesiense (TR) which occurs in Eastern and Southern Africa (Figure 3) [1]. It is transmitted to human beings throughout the biting of the tsetse fly from a reservoir of animal or human virus. Outside these areas, the disease affects travelers who have stayed in Africa. The form due to TG currently counts for $98 \%$ of reported cases of sleeping sickness. The form of the disease due to TR has the particularity to develop more rapidly in comparison with the one caused by TG.

The management of this disease, classified as a neglected tropical disease, is part of a WHO special program which aimed to eradicate it as a public health problem by 2020 and stop its transmission by 2030. Sustained control efforts have reduced the number of new cases. In 2009 the number reported dropped below 10,000 for the first time in 50 years, and in 2019 there were less than 1000 cases recorded [1] [6]. Its diagnosis and treatment require a special care system.

\subsection{Disease History}

HAT due to TG is thus a geographic anthropozoonosis disease that occurs in West and Central Africa [1] [6]. The parasite Trypanosoma brucei gambiense (TG); so named because of its discovery along the Gambia River, was first discovered by Forde in 1901 [4]. It was Dutton who proposed the name Trypanosoma gambiense in 1902. In 1907 the cause-and-effect link between this parasite and sleeping sickness was demonstrated by Gray and Tulloch. The tsetse fly vector of the parasite was identified by Bruce and Nabarro in 1903 and Kleine in 1909. 
Previously, Mott (1899) described the neuropathologic changes in two fatal cases of "Negro Lethargy" or "sleeping sleekness". He described development of diffuse meningoencephalomyelitis involving predominantly the white matter regions. One can also find a perivascular lymphocytic infiltration in the brain and the arachnoid. These descriptions of neuroinflammation are known as the meningoencephalitis stage of the disease. No description revealed signs of hemorrhage [7] [8].

We found in the literature only one clinical study done by Bogaert in 1962, realised on some cats infected for experimentation. Four cats were injected with the parasite. Two died after four months of infection with rapid weight loss, generalized adenopathy and splenomegaly. After 7 months of infection a third cat had a temporary pelvic limb paralysis. At the end of ten months the fourth cat showed severe somnolence, myoclonia, periodic epileptic seizures and severe ataxia of cerebellar type. The necropsy and patho-anatomical data showed cerebral trypanosomiasis: cellular infections of the optic tract, pericapillary infiltrations, uniform meningitis, an infiltration of the optic tracts with significant lesions near the hippocampus and the "ectosylvian" convolution [4] [5].

\subsection{Course of HAT Caused by TG}

The disease operates in stages after the biting of the tsetse fly and leads to sleeping disturbances which characterizes this so-called "sleeping-sickness" [3] [6] [7] [9].

Neuro-ophthalmologic manifestations appear to be important. With regard to the impairment of the visual pathways, Mwanza et al. reported a papillary edema (17\%), cranial nerve palsies (9\%) affecting the oculomotor nerve, the abducens nerve or the facial nerve, the visual field (4\%), a cortical blindness and nystagmus [8] [10]. There was no imaging data in this study.

These data are important and should be taken into account when exploring the optic pathways if encephalitis is suspected, in geographic areas where the vector is present, or when dealing with patients who have travelled in endemic areas.

\subsection{Imaging of HAT and Correlation with Histopathological Animal Data}

High field MRI is the most efficient current technique to explore the nervous system, especially the cranial nerves or optic pathways. Neuroimaging in HAT may be normal or show signs of encephalitis with diffuse vasogenic edema, without hemorrhage or significant contrast enhancement (Figure 2(A), Figure 2(B), Figure 3(A) and Figure 3(B)) in the white matter of the cerebrum and the brainstem, the basal ganglia. This aspect is correlated with Mott's observations (1899). Inflammatory involvement of the optic tracts appears to be characteristic with enlargement, hyperintensity on T2SE and T2 FLAIR sequences and without contrast enhancement (Figure 2(C) and Figure 3(C)) [3] [7]. Correlation with Bo- 
gaert's experimental animal study confirms that this optic tract damage could be a specific sign of meningoencephalitis due to TG [5].

These MRI findings appear to be the first to be correlated with the neuro-experimental data of Mott and Bogaert studies. Therefore, special attention must be paid to the optic tracts, particularly in case of suspected encephalitis in an endemic area or in patients who travelled in it. Conversely, in the event of an incidental discovery, the process of searching trypanosoma in the blood or in cerebrospinal fluid can be launched.

MRI could be integrated into the management process for patients who suffered from HAT. It makes it possible to follow the signal of evolving lesions but also to differentiate melarsoprol-induced encephalitis (arsenical derivative) from those linked to the parasite [2] [7]. In the sense that, arsenical encephalitis causes hemorrhagic lesions while encephalitis THA does not cause hemorrhage.

The article reported by Ugoji is interesting and reassuring with regard to pregnant women. After two years of follow-up, recovery was obtained without sequelae [11].

\subsection{Trypanotolerance}

For many years HAT was considered fatal if untreated but there is growing evidence that some patients affected by TG remain asymptomatic and may self-cure. This is the concept of trypanotolerance. It can explain the relative tolerance against the disease, which can progress very slowly in some people. Sudarshi reported a case of HAT evolving over 29 years.

Other diseases that may or not be fatal can affect patients suffering already from trypanosomiasis. This is what we can learn from trypanotolerance and the possible long course of the disease [3] [9] [12].

\section{Conclusion}

These MRI descriptions on the optic tracts and the encephalitis signs due to TG are in perfect correlation with the animal experimental data carried out by Bogaert in 1962 and Mott's observations in 1899. They seem to be the first described in the reviewed literature. This slowly evolving disease seems to be able to be eradicated. However, vigilance must be constant and MRI must be integrated as a tool for detecting brain damage induced by HAT and as a means of monitoring patients under treatment. This so-called "neglected tropical disease" implies special attention in the WHO eradication program.

\section{Conflicts of Interest}

The authors declare no conflicts of interest regarding the publication of this paper.

\section{References}

[1] Gao, J.-M., Qian, Z.-Y., Hide, G., Lai, D.-H., Lun, Z.-R. and Wu, Z.-D. (2020) Human African Trypanosomiasis: The Current Situation in Endemic Regions and the 
Risks for Non-Endemic Regions from Imported Cases. Parasitology, 147, 922-931. https://doi.org/10.1017/S0031182020000645

[2] Kennedy, P.G.E. (2004) Human African Trypanosomiasis of the CNS: Current Issues and Challenges. The Journal of Clinical Investigation, 113, 496-504.

https://doi.org/10.1172/JCI200421052

[3] Denny, M.C., Lai, L.L. and Laurence, R. (2016) Human African Trypanosomiasis Encephalitis in the United States: Serial Magnetic Resonance Imaging. The Neurohospitalist, 6, 170-173. https://doi.org/10.1177/1941874416637403

[4] Bowman, A. (2014) Trypanosoma Gambiense Dutton, 1902. https://www.aavp.org/wiki/catprotozoa/coccidia-apicomplexan/sarcomastigophora/ trypanosomes-leishmanial-organisms/trypanosoma/trypanosoma-gambiense/

[5] Innes, J.R.M and Saunders, L.Z (1962) Protozoan Infections. In: Comparative Neuropathology, Chapter IX, Academic Press, New York, 473-474.

[6] World Health Organization (2021) Human African Trypanosomiasis. Sleeping Sickness. https://www.who.int/health-topics/human-african-trypanosomiasis\#tab=tab 1

[7] Aidara, C.M., Magne, C., Kouna, P., Mipinda, G.E., Diop, A.D., Diop, A.N., Ba, S. (2017) High Field MRI in Human African Trypanosomiasis (HAT). Open Journal of Radiology, 7, 190-198. https://doi.org/10.4236/ojrad.2017.73021

[8] Rodgers, J., Steiner, I. and Kennedy, P.G.E. (2019) Generation of Neuroinflammation in Human African Trypanosomiasis. Neurology Neuroimmunology \& Neuroinflammation, 6, e610. https://doi.org/10.1212/NXI.0000000000000610

[9] Sudarshi, D., Lawrence, S., Pickrell, W.O., Eligar, V., Walters, R., et al. Human African Trypanosomiasis Presenting at Least 29 Years after Infection-What Can This Teach Us about the Pathogenesis and Control of This Neglected Disease? PLoS Neglected Tropical Diseases, 8, e3349. https://doi.org/10.1371/journal.pntd.0003349

[10] Mwanza, J.C., Kazumba, L., Plant, G.T. and Tylleskär, T. (2004) Manifestations NeuroOphthalmologiques de la Trypanosomiase Humaine Africaine Neuro-Ophthalmologic Manifestations of Human African Trypanosomiasis (English). African Journal of Neurological Sciences, 23. https://doi.org/10.4314/ajns.v23i1.7544

[11] Ugoji, U., Bock-Oruma, A., Umukoro, D. and Ndukwu, G. (2014) Human African Trypanosomiasis Successfully Treated with Melarsoprol in Pregnancy in a Niger Delta Rural Hospital. Case Reports in Clinical Medicine, 3, 353-356. https://doi.org/10.4236/crcm.2014.36078

[12] Bucheton, B., MacLeod, A. and Jeamonneau, V. (2011) Human Host Determinants Influencing the Outcome of Trypanosoma Brucei Gambiense Infections. Parasite Immunology, 33, 438-447. https://doi.org/10.1111/j.1365-3024.2011.01287.x 